\title{
On the Design and Security of RC2
}

Lars R. Knudsen ${ }^{1}$, Vincent Rijmen ${ }^{2}$, Ronald L. Rivest ${ }^{3}$, and Matthew J.B. Robshaw ${ }^{4}$

1 Dept. of Informatics, University of Bergen, Hi-techcenter, N-5020 Bergen, Norway

larsr@ii.uib.no

2 K.U. Leuven, ESAT, Kardinaal Mercierlaan 94, B-3001 Heverlee, Belgium vincent.rijmen@esat.kuleuven.ac.be

3 M.I.T. Laboratory for Computer Science, 545 Technology Square, Cambridge, MA 02139, USA

rivest@theory.lcs.mit.edu

4 RSA Laboratories, 100 Marine Parkway, Redwood City, CA 94065, USA

matt@rsa.com

\begin{abstract}
The block cipher RC2 was designed in 1989 by Ron Rivest for RSA Data Security Inc. In this paper we describe both the cipher and preliminary attempts to use both differential and linear cryptanalysis.
\end{abstract}

\section{Introduction}

RC2 is a block ciphen that was designed in 1989 by Ron Rivest for RSA Data Security, Inc. Initially held as a confidential and proprietary algorithm, RC2 was published as an Internet Draft during 1997 [12. RC2 has many interesting and unique design features, particularly so when one considers the style of ciphers that dominated both the literature and the market at the time of its invention. The cipher was intended to be particularly efficient on 16-bit processors and with a 64-bit block size it was intended as a drop-in replacement for DES [11. A significant feature of $\mathrm{RC} 2$ is the flexibility offered to the user in terms of the effective key-size. This has now become a common feature of many block cipher proposals and it is a property that has proven to be important in commercial applications. Over the years $\mathrm{RC} 2$ has been deployed widely and it features prominently in the S/MIME secure messaging standard 5. Currently there are no published results on the cryptanalytic strength of RC2. As a first step this paper sets out some details on how the basic attacks of differential [1] and linear $[8]$ cryptanalysis might apply.

\section{The Design of RC2}

There are two distinct parts to using RC2. First a key expansion procedure takes a user-supplied key of between one and 128 bytes in length together with a

${ }^{1} \mathrm{RC} 2$ is a registered trademark of RSA Data Security, Inc.

S. Vaudenay (Ed.): Fast Software Encryption - FSE'98, LNCS 1372, pp. 206-221 1998.

(C) Springer-Verlag Berlin Heidelberg 1998 
parameter that specifies the effective key-length of encryption. From this information an array $K[\cdot]$ of 6416 -bit round keys is derived. Then a 64 -bit plaintext block is encrypted using array $K[\cdot]$. Encryption consists of two styles of rounds. One is termed a MIXING round and the other a MASHING round.

Both the key expansion and encryption components rely on the use of a substitution table called PITABLE. This table specifies a random permutation on the integers $0, \ldots, 255$ and was derived from the expansion of $\pi=3.14159 \ldots$. The table itself will not concern us directly in this paper, but it is included for completeness in the Appendix. We will now describe the action of RC2 in more detail. We will use $x<<<k$ to denote the 16-bit word $x$ rotated left by $k$ bits, $\&$ will denote bitwise logical AND, $\oplus$ will denote bitwise exclusive-or and $~$ will denote bitwise complementation. All 16-bit word addition + is performed modulo $2^{16}$.

\subsection{Key Expansion}

During the key expansion procedure both byte operations and 16-bit word operations are used. The array $K[\cdot]$ that stores the 6416 -bit round keys will be referred to in two ways.

a) For word operations the positions of the buffer will be referred to as $K[0]$, $\ldots, K[63]$ where each $K[i]$ is a 16-bit word.

b) For byte operations the array of round keys will be referred to as $L[0], \ldots$, $L[127]$ where each $L[i]$ is an eight-bit byte. It will always be the case that $K[i]=L[2 i]+256 \times L[2 i+1]$ (That is, the lower order byte is given first).

Suppose that $T$ bytes of key are supplied by the user with $1 \leq T \leq 128$. The key expansion procedure places the $T$-byte key into $L[0], \ldots, L[T-1]$ of the key buffer. Regardless of the value of $T$ however, the algorithm has a maximum effective key length in bits that is denoted $T 1$. The effective key length in bytes $T 8$ and a mask $T M$ based on the effective key length in bits $T 1$ are derived as $T 8=\lceil T 1 / 8\rceil$ and $T M=255 \bmod 2^{8(1-T 8)+T 1}$. Key expansion consists of the following two loops and intermediate step:

1. for $i=T, T+1, \ldots, 127$ do

$L[i]=\operatorname{PITABLE}[L[i-1]+L[i-T]]$ (addition is modulo 256)

2. $L[128-T 8]=$ PITABLE $[L[128-T 8] \& T M]$

3. for $i=127-T 8, \ldots, 0$ do

$$
L[i]=\operatorname{PITABLE}[L[i+1] \oplus L[i+T 8]]
$$

At the end of this key expansion the array $K[0], \ldots, K[63]$ contains the 64 16-bit subkey words that will be used during encryption.

\subsection{Encryption and Decryption}

The encryption operation is defined in terms of primitive MIX and MASH operations. An array of four 16-bit words $R[0], \ldots, R[3]$ are used to hold the initial plaintext, the intermediate results, and the final ciphertext. Indices to this array are always given modulo 4 . 
$\operatorname{MIX} R[i]$

The primitive "MIX $R[i]$ " operation is defined as follows, where $s[0]=1$, $s[1]=2, s[2]=3$, and $s[3]=5$. Here $j$ is a "global" variable so that $K[j]$ is always the first key word in the expanded key which has not yet been used in a MIX operation.

$$
\begin{aligned}
& R[i]=R[i]+K[j]+(R[i-1] \& R[i-2])+(\sim R[i-1] \& R[i-3]) \\
& j=j+1 \\
& R[i]=R[i]<<<s[i]
\end{aligned}
$$

MIXING round

A MIXING round consists of MIX $R[0]$, MIX $R[1]$, MIX $R[2]$, MIX $R[3]$. MASH $R[i]$

The primitive "MASH $R[i]$ " operation is defined as follows:

$$
R[i]=R[i]+K\left[R[i-1] \& 003 \mathbf{f}_{\mathbf{x}}\right]
$$

\section{MASHING round}

A MASHING round consists of MASH $R[0]$, MASH $R[1]$, MASH $R[2]$, MASH $R[3]$.

The entire encryption operation can now be described as follows. Here $j$ is a global integer variable which is only affected by the mixing operations.

Encryption With RC2

1. Initialize words $R[0], \ldots, R[3]$ to contain the 64 -bit plaintext block.

2. Expand the key, so that words $K[0], \ldots, K[63]$ become defined.

3. Initialize $j$ to zero.

4. Perform five MIXING rounds.

5. Perform one MASHING round.

6. Perform six MIXING rounds.

7. Perform one MASHING round.

8. Perform five MIXING rounds.

9. The ciphertext is $R[0], \ldots, R[3]$.

Decryption is the reverse of encryption. Since the details can easily be established they are not included here. Test vectors for encryption using RC2 are provided in the Appendix.

\subsection{Features of RC2}

$\mathrm{RC} 2$ is rather unusual in that the 64-bit plaintext block is split into four words each of 16 bits. In a style reminiscent of the hash function MD4 [13], much of the encryption process relies on one of these words being modified by a function of the other three, the four words then being swapped cyclically. This design 
approach was explored some seven years after the design of $\mathrm{RC} 2$, which now might be described as being an "unbalanced Feistel cipher" 4 .

The key schedule for RC2 is also unusual. $T 8$ is the number of bytes needed to contain the given $T 1$ bits of key. When $T 1$ is congruent to $k$, modulo 8 , a mask $T M$ containing ones in the low-order $k$ bits is used to derive the correct effective key length. The first step of the key expansion expands the key to a full 128 bytes, using a non-linear byte-wide feedback shift-register approach. Step three is similar to the first, except that it starts at the high end and works towards the lower end. Steps two and three also work together to limit the effective key size to $T 1$ bits. Step three corresponds to using a feedback register of only T8 bytes, and step two ensures that the initial state of that register has only $T 1$ bits of entropy. Although the procedure limits the actually entropy of the key to $T 1$ bits, it also ensures that the final key table depends upon each bit of the supplied key. If one supplies a 16 -byte key, but set $T 1=40$, then changing any bit of the supplied key should result in a different key table, although the number of possible key tables is limited to $2^{40}$.

\section{Differential Cryptanalysis of RC2}

Differential cryptanalysis [1] can be a powerful style of attack. By choosing a pair of plaintexts with a particular difference, which can be adapted to the cipher in question, the cryptanalyst hopes that some identifiable, and unusual behavior, can be observed by processing the ciphertexts. One possible evolution of the difference between a pair of plaintexts during encryption can be described by a characteristic. In essence, a characteristic specifies the difference between two parallel encryptions at each stage of the encryption process and there is some associated probability that a pair being encrypted does indeed follow this description. A plaintext pair that follows the characteristic is typically called a right pair. A pair that does not is called a wrong pair.

Throughout our attack on $\mathrm{RC} 2$, we shall define the difference between two 16-bit words $A$ and $B$ to be $A \oplus B$. Furthermore in our analysis we shall be interested in how single-bit differences behave within RC2. The decision to restrict our attention to single-bit differences facilitates analysis but is also motivated by a typical assumption that characteristics involving multiple-bit differences over integer addition will generally hold with lower probability than single-bit characteristics [6]. We note that other more complex techniques [2]7] might open new avenues for the analysis of $\mathrm{RC} 2$.

We will use $e_{t}$ to denote the 16 -bit word with a single one bit in position $t$ from the right, all other bits being set to zero. We also view the leftmost bit of a 16-bit word to be the most significant bit. Thus we shall use $e_{15}$ to denote a 16bit word with the only non-zero bit being the most significant bit. We will denote the word of 16 zero bits as $0000 \mathrm{x}$ where the subscript $\mathrm{x}$ denotes hexadecimal notation and we will denote the Hamming weight (i.e. the number of ones in the binary expansion of some quantity $x)$ as $\operatorname{Hwt}(x)$. 
For the remainder of the paper, we shall consider MIXING and MASHING rounds in the following way. Instead of viewing the operation at each step as acting on a different word we shall consider the operations to be identical (i.e., at each MIX step $R[0]=R[0]+K[j]+(R[3] \& R[2])+(\sim R[3] \& R[1]))$ but that between steps the words are rotated cyclically (i.e., TEMP $=R[0] ; R[0]=R[1] ; R[1]=R[2]$; $R[2]=R[3] ; R[3]=\mathrm{TEMP})$.

\subsection{Some Basic Characteristics for MIX}

Given an input difference $\left(e_{t} 0000_{\mathrm{X}} 0000_{\mathrm{X}} \quad 0000_{\mathrm{X}}\right)$ to the first MIX step in a MIXING round, the output difference before rotation will be $\left(e_{t} 0000_{\mathrm{x}} 0000_{\mathrm{x}}\right.$ $0000_{\mathrm{X}}$ ) with probability $p \geq 1 / 2$. Rotation then moves this single bit difference within the word, and the four words are swapped cyclically. We can summarize the four basic characteristics which hold with probability $p \geq 1 / 2$ (when averaged over all plaintexts and key words) for a MIX step. The value of the rotation $s[i]$ depends on the step $i$ in which the characteristic is applied. Note that addition within the subscript of $e_{t}$ is to be performed modulo 16 .

$$
\begin{aligned}
& \left(e_{t} 0000_{\mathrm{x}} 0000_{\mathrm{x}} 0000_{\mathrm{x}}\right) \rightarrow\left(0000_{\mathrm{x}} 0000_{\mathrm{x}} 0000_{\mathrm{x}} e_{t+s[i]}\right) \\
& \left(0000_{\mathrm{x}} 0000_{\mathrm{x}} 0000_{\mathrm{x}} e_{t}\right) \rightarrow\left(0000_{\mathrm{x}} 0000_{\mathrm{x}} e_{t} 0000_{\mathrm{x}}\right) \\
& \left(0000_{\mathrm{x}} 0000_{\mathrm{x}} e_{t} 0000_{\mathrm{x}}\right) \rightarrow\left(0000_{\mathrm{x}} e_{t} 0000_{\mathrm{x}} 0000_{\mathrm{x}}\right) \\
& \left(0000_{\mathrm{x}} e_{t} 0000_{\mathrm{x}} 0000_{\mathrm{x}}\right) \rightarrow\left(e_{t} 0000_{\mathrm{x}} 0000_{\mathrm{x}} 0000_{\mathrm{x}}\right)
\end{aligned}
$$

Apart from (1) with $t=15$ which holds with probability $p=1$, these characteristics hold with probability $p=1 / 2$ on average. There are times where the characteristics do not hold. The following are the cases where the characteristic hold with certainty:

- In $(2)$, if $\left(R[2] \& e_{t}\right)=\left(R[1] \& e_{t}\right)$ then $p=1$.

- In $(3)$, if $\left(R[3] \& e_{t}\right)=0000_{\mathbf{x}}$ then $p=1$.

- In (4), if $\left(R[3] \& e_{t}\right)=e_{t}$ then $p=1$.

In the first MIXING round, the attacker chooses the plaintext and this allows the cryptanalyst to capture some of these special cases in an attack.

\subsection{Some Basic Characteristics for MASH}

There are two MASHING rounds in RC2 and the basic MASH step is $R[0]=$ $R[0]+K\left[R[3] \& 003 \mathrm{f}_{\mathrm{x}}\right]$. Given an input difference $\left(0000_{\mathrm{x}} 0000_{\mathrm{x}} 0000_{\mathrm{x}} e_{t}\right)$ to a MASHING round with $\left(e_{t} \& 003 \mathrm{f}_{\mathrm{x}}\right)=0000_{\mathrm{x}}$ the same key word $K[\cdot]$ will be added to both sets of partially encrypted data. The four basic useful characteristics for MASH are as follows:

$$
\begin{aligned}
& \left(e_{t} 0000 \mathrm{x} 0000 \mathrm{x} 0000 \mathrm{x}\right) \rightarrow\left(0000 \mathrm{x} 0000_{\mathrm{x}} 0000_{\mathrm{x}} e_{t}\right) \\
& \left(0000 \mathrm{x} 0000_{\mathrm{x}} 0000_{\mathrm{x}} e_{t}\right) \rightarrow\left(0000 \mathrm{x} 0000 \mathrm{x} e_{t} 0000 \mathrm{x}\right) \\
& \left(0000_{\mathrm{x}} 0000_{\mathrm{x}} e_{t} 0000_{\mathrm{x}}\right) \rightarrow\left(0000_{\mathrm{x}} e_{t} 0000_{\mathrm{x}} 0000_{\mathrm{x}}\right) \\
& \left(0000_{\mathrm{x}} e_{t} 0000_{\mathrm{x}} 0000_{\mathrm{x}}\right) \rightarrow\left(e_{t} 0000_{\mathrm{x}} 0000_{\mathrm{x}} 0000_{\mathrm{x}}\right)
\end{aligned}
$$


Characteristic (5) holds with probability $p=1 / 2$ unless $t=15$ when it holds with probability $p=1$, characteristics (7) and (8) hold with probability $p=1$, and characteristic (6) holds with probability $p=1$ if $\left(e_{t} \& 0 \mathrm{x} 3 \mathrm{f}\right)=0000_{\mathrm{x}}$. Joining these four characteristics together to pass across a MASHING round with probability $p=1$ is straightforward.

\subsection{Towards a Differential Attack on RC2}

In this section we combine characteristics for both MIXING and MASHING rounds while moving towards a full analysis of RC2. We will assume that the subkey words $K[0], \ldots, K[63]$ are independent and we aim to recover the expanded key table $K[\cdot]$ in our attack.

The characteristics of interest are built around single-bit differences and as noted in Section 3.1 there are advantages to having this single non-zero bit in the most significant bit of a word. Depending on which word $R[\cdot]$ is the subject of the characteristic we use, different rotation amounts feature during MIXING. This leads to conditions on $t$, the position of the single-bit difference in the plaintext, that provide some advantages in an attack. Another consideration is the presence of the MASHING rounds and one aim might be to nullify their action. If a one-bit characteristic specifies an input difference to a MASHING round of $e_{t}$ in any one of the words, then provided $t=15$ the characteristic will pass through the MASHING round unhindered with probability $p=1$. If $5<t<15$ then there is a characteristic that holds with probability $p=1 / 2$. There are six MIXING rounds between the two MASHING rounds and so with the difference $\left(e_{t}\right.$ $0000_{\mathrm{X}} 0000_{\mathrm{X}} 0000_{\mathrm{x}}$ ) as input to the first MASHING round we can establish the values of $t$ that are useful to us.

A more accurate reflection of the success of a final attack is given by considering differentials [10] instead of characteristics (which provide only a lower bound to the probability of the differential). In Section 3.5 we will consider the issue of differentials in more detail but from this point on we will anticipate later analysis by referring to the use of differentials during our description of the attack. The observations provided so far allow us to present in Table 1 the differentials that are useful to us.

\subsection{Recovering Key Information}

In a differential cryptanalytic attack the attacker typically chooses a differential for $(n-1)$ rounds of an $n$-round block cipher. The attacker then tries to deduce key information from the last round of the cipher 1 ]. Here, the most effective attack on RC2 appears to require that bits of the subkey $K[0]$ used in the first MIXING round are recovered first.

Consider a differential with input difference $\left(0000_{\mathrm{x}} \quad 0000_{\mathrm{x}} \quad 0000_{\mathrm{x}} e_{t}\right)$. The starting values of $R[1]$ and $R[2]$ are chosen so that $\left(R[1] \& e_{t}\right)=\left(R[2] \& e_{t}\right)$. After the first MIX step the difference will be $\left(0000_{\mathrm{x}} 0000_{\mathrm{x}} e_{t} 0000_{\mathrm{x}}\right)$. The output difference from the second MIX step will depend on the value of bit $t$ in register $R[3]$. If this bit is zero then word $R[1]$ with difference $0000_{\mathrm{x}}$ will be 


\begin{tabular}{|c|c|c|c|}
\hline ext differ & $\begin{array}{l}\text { ce at start of } \\
\text { XING round }\end{array}$ & prob. & alu \\
\hline$t_{t} 0000 \mathrm{x} \quad 0000 \mathrm{x}$ & $\left(e_{t+15} 0000 \mathrm{x} \quad 0000 \mathrm{x} 000\right.$ & & 4 \\
\hline$\left.e_{t} \quad 0000_{\mathrm{x}} \quad 0000_{\mathrm{x}} \quad 0000_{\mathrm{x}}\right)$ & $\left(\begin{array}{llll}e_{t+15} & 0000_{\mathrm{x}} & 0000_{\mathrm{x}} & 000\end{array}\right.$ & & 1,2 , \\
\hline $0000 \mathrm{x}$ et $\left.\quad e_{t} 0000 \mathrm{x} \quad 0000_{\mathrm{x}}\right)$ & $\left(0000 \mathrm{x} \quad e_{t+14} 0000 \mathrm{x} \quad 0000_{\mathrm{x}}\right)$ & & 5 \\
\hline$\left(\begin{array}{llll}0000_{\mathrm{x}} & e_{t} & 0000_{\mathrm{x}} & 0000_{\mathrm{x}}\end{array}\right)$ & $\left(0000_{\mathrm{x}} e_{t+14} 0000_{\mathrm{x}} \quad 0000_{\mathrm{x}}\right)$ & & 1, \\
\hline$\left(0000 \mathrm{x} \quad e_{t} \quad 0000 \mathrm{x} \quad 0000 \mathrm{x}\right)$ & $\left(0000 \mathrm{x} \quad e_{t+14} 0000 \mathrm{x} \quad 0000_{\mathrm{x}}\right)$ & & $, 2,4$ \\
\hline $\left.0000 \mathrm{x} \quad 0000 \mathrm{x} \quad e_{t} 0000 \mathrm{x}\right)$ & $\left(\begin{array}{llll}0000 \mathrm{x} & 0000 \mathrm{x} & e_{t+13} & 0000 \mathrm{x}\end{array}\right)$ & & 14 \\
\hline$\left(\begin{array}{llll}0000_{\mathrm{x}} & 0000_{\mathrm{x}} & e_{t} & 0000_{\mathrm{x}}\end{array}\right)$ & $\left(\begin{array}{llll}0000 \mathrm{x} & 0000_{\mathrm{x}} & e_{t+13} & 0000_{\mathrm{x}}\end{array}\right)$ & 2 & $7, \ldots, 13$ \\
\hline$\left(\begin{array}{llll}0000 \mathrm{x} & 0000 \mathrm{x} & 0000 \mathrm{x} & e_{t}\end{array}\right)$ & $\left(\begin{array}{llll}0000 \mathrm{x} & 0000 \mathrm{x} & 0000 \mathrm{x} & e_{t+11}\end{array}\right)$ & 2 & 6 \\
\hline $0000_{\mathrm{x}} \quad 0000_{\mathrm{x}} 00$ & $0_{x} \quad 0000_{x} \quad 0000_{x}$ & & 0, \\
\hline
\end{tabular}

Table 1. 26 differentials that are potentially useful in an attack on RC2. The associated probabilities are lower bounds provided by the analysis of a characteristic contained within the specified differential.

chosen. Otherwise word $R[2]$ with difference $e_{t}$ will be selected and a difference will be introduced into another word. Note that the value of this bit depends on the plaintext (which we know) and on bits of the first 16-bit subkey word $K[0]$.

We can trace the output of the second MIX step to the end of the penultimate MIXING round by using the differentials in Table 1 If the pair is a right pair then we can recover one bit of information from $K[0]$ as follows. A necessary condition for a pair to be a good pair is that

$$
\begin{aligned}
e_{t} & \&((R[0]+(R[3] \& R[2]) \\
& +(\sim R[3] \& R[1])+K[0])<<<1)=0 .
\end{aligned}
$$

Let $x=R[0]+(R[3] \& R[2])+(\sim R[3] \& R[1])$ which we control via the choice of plaintext. Then we have the following condition for a right pair:

$$
(x+K[0]) \& e_{t-1}=0 .
$$

Denote by $k$ the value derived by setting the top $((16-t) \bmod 16)$ bits of $K[0]$ to zero. Let $y=x \& e_{t-1}$ and let $z$ be the quantity derived by setting the top $((16-t+1) \bmod 16)$ bits of $x$ to zero. Then we have that

$$
(x+K[0]) \& e_{t-1}=0 \Leftrightarrow y=(z+k) \& e_{t-1} .
$$

To mount an attack to recover bit $(t-1)$ of $k$ for some given $t$ we encrypt plaintext pairs with $z=0$ until we obtain a right pair. Once we have a right pair we observe the value of $y$. From this we deduce the value of bit $(t-1)$ in $k$ and hence in $K[0]$. We can then repeat this approach choosing pairs with different values to $z$ so that information on the subkey $K[0]$ is recovered bit by bit.

By using different differentials with different values of $t$ (see Table 1) we are able to introduce some error-checking into the attack 2 . In this way the bits of

\footnotetext{
${ }^{2}$ Not all values of $t$ are valid for use due to the two MASHING rounds.
} 
$K[0]$ that we recover can be verified. All recovered bits of $K[0]$ have to be correct before the next bit of $K[0]$ can be correctly derived. Note that structures [1] can be useful in reducing the plaintext requirements for a differential attack when more than one differential is useful. With $n$ useful differentials we can ask for a structure of $2^{n}$ plaintexts with specifically chosen differences. From these we derive $2^{n-1}$ plaintext pairs for each of the $n$ characteristics.

There remains the issue of detecting when a data pair is a good pair. We note that the difference at the start of the final MIXING round has Hamming weight one for a good pair. We might therefore measure the Hamming weight of the ciphertext and if the weight is less than some threshold the pair can be considered a right pair. Depending on the threshold we might accept some wrong pairs as being right pairs, something that would provide a wrong answer to the bit we wish to recover with probability $1 / 2$. To improve the robustness of the attack one might aim to collect more right pairs. Then the value of the bit suggested most often can be assumed to be the correct value to the key bit we are trying to recover. As a demonstration we provide the success rate for different amounts of plaintext in experiments on eight-round RC2. (There are eight MIXING rounds with a MASHING inserted after round five as occurs in RC2.) A decision on whether a good pair had occurred was made according to whether the Hamming weight of the difference in the ciphertext was less than some threshold. Then, once a value for the key bit had been counted more than the other (this difference being denoted by excess) that value for the key bit was set. Each entry in the table was computed after 20 experiments.

\begin{tabular}{|c|rr|rl|rl|}
\hline & \multicolumn{5}{|c|}{ Hamming weight } \\
excess & \multicolumn{1}{|c|}{11} & \multicolumn{2}{c|}{13} \\
\hline 2 & $90 \%$ & $2^{29}$ & $20 \%$ & $2^{28.5}$ & $0 \%$ & $2^{27.5}$ \\
4 & $100 \%$ & $2^{30}$ & $95 \%$ & $2^{30}$ & $20 \%$ & $2^{29.5}$ \\
8 & $100 \%$ & $2^{31}$ & $100 \%$ & $2^{31}$ & $65 \%$ & $2^{31}$ \\
\hline
\end{tabular}

\subsection{The Effectiveness of Differential Cryptanalysis}

As we previously mentioned it is differentials and their probabilities that reflect the effectiveness of a differential attack. Whereas a characteristic describes one specific evolution of differences through encryption, from a given starting difference there might well have been other "paths" through the cipher to the same target difference than the one described by one particular characteristic. With RC2 this leads to a particularly interesting interaction between the MIXING and MASHING rounds.

First we will consider in abstract terms the probability that a one-bit difference in some word $a$ produces a one-bit difference in the word $d$ when we define $d=a+b+c$ for unknown constants $b$ and $c$. One approach might be to consider this as two separate additions and to consider the intermediate word $e=a+b$ first. Since a one-bit difference in $a$ produces a one-bit difference in $e$ with probability $1 / 2$ and a one-bit difference in $e$ provides a one-bit difference in $d=e+c$ with probability $1 / 2$ we would say that the characteristic over the two 
additions has probability $1 / 4$. However it would then be misleading to use this characteristic to provide an approximation to the probability of the differential from $a$ to $d$. Instead, the probability of the propagation of a one-bit difference from $a$ to $d$ is $1 / 2$ since $b+c$ is a fixed value. Consequently the probability of the differential from $a$ to $d$ must also be $1 / 2$.

Recall that the probability of the differential is given by the sum of the probabilities of all the characteristics that satisfy the differential. By looking at two successive additions in isolation we inadvertently restrict our attention to single-bit differences in the intermediate value $e$. Let $\alpha, 0 \leq \alpha \leq n-1$, denote the position of the one bit difference in $a$. A one-bit difference in $a$ will give a difference in $e$ with Hamming weight $h$ with probability $2^{-h}, 1 \leq h<n-\alpha$, and with probability $2^{-n+\alpha+1}$ for $h=n-\alpha$. Since this $h$-bit difference was caused by a one-bit difference in the previous step 3 an $h$-bit difference in $e$ will be transformed to a one-bit difference in $d$ by the addition of $c$ with probability $1 / 2$. Thus we get

$$
\begin{aligned}
& p=2^{-1}\left(2^{-n+\alpha}+\sum_{h=1}^{n-\alpha} 2^{-h}\right), \quad \text { if } \alpha<n-1 \\
& p=1 \text { if } \alpha=n-1 .
\end{aligned}
$$

One place where this has an effect is when a MIXING round follows a MASHING round. Each word $R[0], \ldots, R[3]$ is modified by a MASH step in turn. At the first subsequent MIX step $R[0]$ is modified by means of addition. By looking at the two additions in isolation one under-estimates the probability of the differential.

In the analysis of $\mathrm{RC} 2$ we need to take account of this effect since it applies to some extent to the MIXING rounds as well as during the transition between MIXING and MASHING rounds. Within the MIXING rounds an intermediate quantity is used as input to a multiplexor function. This reduces the probability that this particular characteristic is followed by a factor of $2^{-h}$ for each multiplexor when the Hamming weight of the difference is $h$. If we denote the number of multiplexing functions between two successive additions by $k$ then (12) can be rewritten as follows:

$$
\begin{aligned}
p & =\sum_{h=1}^{n-\alpha} 2^{-h} \cdot 2^{-h k} \cdot 2^{-1}+2^{-(n-\alpha)} \cdot 2^{-(n-\alpha) k} \cdot 2^{-1} \\
& =2^{-1}\left(\sum_{h=1}^{n-\alpha} 2^{-(k+1) h}+2^{-(k+1)(n-\alpha)}\right) \\
& =2^{-1}\left(\frac{1-2^{-(k+1)(n-\alpha)}}{1-2^{-(k+1)}} \frac{1}{2^{(k+1)}}\right)+2^{-(k+1)(n-\alpha)} \\
& \approx 2^{-1}\left(\frac{1}{2^{(k+1)}-1}\right)
\end{aligned}
$$

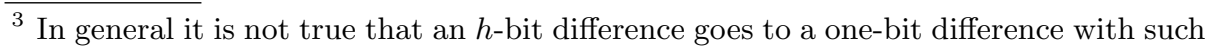
a high probability. 
The last approximation is reasonable for smaller $\alpha(\alpha<n-3)$ but would need some correction for larger values of $\alpha$. For $k=0,1,2,3$, (17) gives $p=$ $1 / 2,1 / 6,1 / 14,1 / 30$, which should be compared with the respective probabilities of the characteristics we previously derived: $1 / 4,1 / 8,1 / 16,1 / 32$. In the case of two consecutive MIXING rounds we have that $k=3$ and so the probability of a one-bit to one-bit differential across two MIXING rounds is $1 / 30 \times 2^{-3}=1 / 240$.

The effect we are using here can be extended to a series of additions whereby the intermediate values of interest have differences with a variety of Hamming weights even though the starting and ending difference have weight one. Consider three consecutive mixing rounds. Let $a$ be a one-bit difference in the leftmost words of two inputs and let $\alpha$ be the position of that bit, where $0 \leq \alpha \leq n-1$. Let $d$ be the difference in the leftmost words after three mixing rounds and suppose that $h_{1}$ and $h_{2}$ denote the Hamming weights of the leftmost words after one, respectively two, mixing rounds. Then the probability that $d$ is a one-bit difference can be estimated as follows, where $k=3$ and where for simplicity we have eliminated the term for $h=n-\alpha$.

$$
\begin{aligned}
p & \simeq \sum_{h_{1}=1}^{n-\alpha} \sum_{h_{2}=1}^{n-\alpha} 2^{-h_{1}} \cdot 2^{-h_{1} k} \cdot 2^{-h_{2}} \cdot 2^{-h_{2} k} \cdot 2^{-1} \\
& =2^{-1}\left(\sum_{h_{1}=1}^{n-\alpha} 2^{-(k+1) h_{1}} \cdot \sum_{h_{2}=1}^{n-\alpha} 2^{-(k+1) h_{2}}\right) \\
& \approx 2^{-1}\left(\frac{1}{2^{(k+1)}-1}\right)\left(\frac{1}{2^{(k+1)}-1}\right) .
\end{aligned}
$$

Again the final approximation requires that $\alpha$ is small. For $k=3 p$ is $2^{-1}(1 / 15)^{2}$. We can now estimate the probability of the differential over three mixing rounds by $2^{-1}(1 / 15)^{2} \times 1 / 8 \simeq 1 / 3600$. This extends easily to more rounds and in general the probability of a differential over $r$ mixing rounds is $(1 / 15)^{r-1} \times 1 / 16$. Note that the MASHING rounds can be passed with probability one.

For a more accurate assessment a slight correction should be applied for rounds where the difference is close to the most significant bit, but experimental evidence given below suggests that the expressions derived are reasonable to use. The number of rounds in the table refers to the number of MIXING rounds used. After five MIXING rounds an additional MASHING round is inserted as occurs when encrypting with RC2. The final column is derived as an average over at least five sets of experiments for each row.

\begin{tabular}{|c|c|c|c|}
\hline \# rounds & \# pairs/test & \# right pairs expected & \# right pairs obtained \\
\hline 3 & $2^{19}$ & 146 & 146 \\
4 & $2^{22}$ & 78 & 79 \\
6 & $2^{29}$ & 44 & 47 \\
7 & $2^{31}$ & 12 & 13 \\
\hline
\end{tabular}

Note that the probability of the differential obtained in this section does not take into account text pairs which have internal differences in more than one word before they resynchronize. This was observed occasionally during experiments 
but cases where differences in more than one word resynchronize are rare and we ignore their impact on our estimates.

\subsection{Differential Cryptanalysis of RC2}

We arrive at the following estimates for the data required to recover information about the subkey $K[0]$. Once this subkey word has been recovered the attack is repeated on what would now become a reduced version of $\mathrm{RC} 2$. When we take into account the key-recovery techniques of Section 3.4 we estimate that a differential attack on RC2 with $r$ MIXING rounds (including the MASHING rounds) requires at most $2^{4 r}$ chosen plaintexts. An attack on RC2 with 16 MIXING rounds requires use of a differential with probability at least $2^{-58.7}$ ("at least" since we have not yet accounted for such phenomena as a one-bit difference in the most significant bit at a MIXING round). In this regard RC2 with 16 MIXING rounds compares favorably to DES ( $2^{47}$ pairs [1]) and 12-round RC5 (244 pairs [3]). It is fair to observe, however, that $\mathrm{RC} 2$ is not a fast cipher and an optimized version of DES and 12-round RC5 are both likely to be faster than RC2.

\section{Linear Cryptanalysis of RC2}

Linear cryptanalysis has provided the best theoretical attack on DES in terms of data requirements [9. However, its usefulness on other ciphers is often limited. The aim of such an attack is to relate bits of the plaintext and ciphertext to bits of the key via a linear equation which holds with some probability $p$. Such an approximation can generally be used to provide an estimate for one bit of the key and more advanced techniques are available to extract more key information 9 . If an approximation holds with probability $p$ then the important quantity for the cryptanalyst is the absolute value of the bias of the approximation $b=|p-1 / 2|$. Typically the data required to use such an approximation is given by $c \times b^{-2}$ known plaintexts for some small constant $c$ [9].

The MIX step in RC2 is $R[0]=R[0]+K[j]+(R[3] \& R[2])+(\sim R[3] \& R[1])$. Across integer addition the best linear approximation involves the least significant bit of each quantity, and will hold with probability one. The multiplexor function $x=(R[3] \& R[2])+(\sim R[3] \& R[1])$ has linear approximations of varying usefulness. The absolute value of the highest non-trivial bias is $1 / 4$ when averaged over all plaintexts. In a slight abuse of notation we will consider a 16-bit word $x$ as a vector in $\mathrm{Z}_{2}^{16}$ and we will use the 16-bit quantity $\alpha$ to indicate the bits of $x$ that are to be used in a linear approximation. This is most conveniently described by means of the scalar product of two vectors. Thus the $\{0,1\}$-vector $\alpha$ will be used to denote the specific bits of $x$ to be used in an approximation and $\alpha \cdot x$ is the value of these bits combined using exclusive-or. Useful linear approximations across the multiplexor are of the form

$$
\begin{array}{ll}
\alpha \cdot x=\alpha \cdot R[1] & \alpha \cdot x=\alpha \cdot R[1] \oplus \alpha \cdot R[3] \\
\alpha \cdot x=\alpha \cdot R[2] & \alpha \cdot x=\alpha \cdot R[2] \oplus \alpha \cdot R[3]
\end{array}
$$


where $\operatorname{Hwt}(\alpha)=1$. More generally approximations to the multiplexor function with non-zero bias have the form

$$
\delta \cdot x=\alpha \cdot R[1] \oplus \beta \cdot R[2] \oplus \gamma \cdot R[3]
$$

where $\delta$ is the bitwise inclusive-or of $\alpha$ and $\beta$ and $\gamma$ is either 0 or it consists of ones in positions where either $\alpha$ or $\beta$ have ones. The greater the value of $\operatorname{Hwt}(\gamma)$ the lower the absolute value of the bias of the approximation.

The following approximation to the first MIX step (which includes the cyclic swap of the $R[\cdot]$ words) might be useful

$$
e_{1} \cdot\left(R[3]^{\text {new }}\right)=e_{0} \cdot\left(R[0]^{\text {old }}\right) \oplus e_{0} \cdot(K[j]) \oplus e_{0} \cdot R[2]^{\text {old }} .
$$

This has a bias of absolute value $1 / 4$. The following steps require no approximation and there appears to be no better non-trivial linear approximations for a complete MIXING round. We might illustrate this approximation in the following way:

$\begin{array}{cccccc}\text { step } & R[0] & R[1] & R[2] & R[3] & \text { round 1 } \\ & e_{0} & - & e_{0} & - & \text { start } \\ 1 & - & - & - & e_{1} & |b|=1 / 4 \\ 2 & - & - & e_{1} & - & |b|=1 / 2 \\ 3 & - & e_{1} & - & - & |b|=1 / 2 \\ 4 & e_{1} & - & - & - & |b|=1 / 2\end{array}$

In continuing this approximation into the next MIXING round we would be forced to approximate the bit $e_{1} \& R[0]$. One integer addition involves the subkey word $K[4]$ and depending on this value the bias of the approximation will vary 4 . The second integer addition involves the output from the multiplexor function. By the conditions given above this approximation must involve $e_{1} \& R[1]$ or $e_{1} \& R[2]$ and we can construct the following approximations for the second and third MIXING rounds. Here we assume that the bias of the approximation across the multiplexor function is at most $1 / 4$. Similarly, we assume that the bias of the approximation across the integer addition is at most $1 / 4$. This occurs in approximating steps 1 and 3 and the value of $|b|$ is given for those steps individually.

$\begin{array}{cccccc}\text { step } & R[0] & R[1] & R[2] & R[3] & \text { round 2 } \\ & e_{1} & - & - & - & \text { start } \\ 1 & - & e_{1} & - & e_{2} & |b| \leq 1 / 8 \\ 2 & e_{1} & - & e_{2} & - & |b|=1 / 2 \\ 3 & - & e_{1} \oplus e_{2} & - & e_{4} & |b| \leq 1 / 8 \\ 4 & e_{1} \oplus e_{2} & - & e_{4} & - & |b|=1 / 2\end{array}$

\subsection{The Effectiveness of Linear Cryptanalysis}

The typical way to measure the effectiveness of linear cryptanalysis is to appeal to the so-called piling-up lemma [8]. By doing this, we are lead to estimate a bias

\footnotetext{
${ }^{4}$ Note that the whole issue of key-dependence in linear cryptanalysis is a complex one that is rarely addressed in detail.
} 
of $\leq 2^{-2} \times 2^{-3} \times 2^{-3} \times 2^{2}=2^{-6}$ for our approximation to the first two MIXING rounds of $\mathrm{RC} 2$. In the case of $\mathrm{RC} 2$, however, routine use of the piling-up lemma can lead to misleading results.

As an example, suppose that the two subkeys used in steps one and three of round two are zero. In isolation the approximation to step one ( $A_{1}$, say) holds with probability $5 / 8$. In step three we find that the second approximation $\left(A_{2}\right.$, say) involves bits that previously determined whether $A_{1}$ held. Analysis shows that the probability that $A_{2}$ holds given that $A_{1}$ held is $13 / 20$ and not $5 / 8$ when $A_{2}$ is considered in isolation. Furthermore, the probability that $A_{2}$ doesn't hold when $A_{1}$ doesn't hold is $5 / 12$ instead of $3 / 8$. So when the two approximations are combined the probability that the combined approximation to round two holds is $(5 / 8 \times 13 / 20)+(3 / 8 \times 5 / 12)=9 / 16$ which leads to a bias of $1 / 16$. This is greater than the $1 / 32$ predicted by use of the piling-up lemma.

Much of the complicated interaction between the two approximations is due to the role of addition in the cipher. As an example, if we suppose that approximation $A_{1}$ holds, then it can be shown that the probability that the least significant bit of $R[2]$ is equal to zero is $11 / 20$. Since this bit plays a pivotal role in determining whether $A_{2}$ holds it is no surprise that the piling-up lemma gives misleading results.

For the user of RC2 there is circumstantial evidence that linear cryptanalysis is unlikely to pose a threat to $\mathrm{RC} 2$. Such attacks appear to be ineffective for ciphers that mix integer addition and bitwise operations unless the approximation can be limited to the least significant bits across an addition [6]. Such a restriction appears unlikely as an extension of the current approximation into a third MIXING round illustrates:

$\begin{array}{cccccc}\text { step } & R[0] & R[1] & R[2] & R[3] & \text { round 3 } \\ & e_{1} \oplus e_{2} & - & e_{4} & - & \text { start } \\ 1 & - & e_{1} \oplus e_{2} \oplus e_{4} & - & e_{2} \oplus e_{3} & |b| \leq 1 / 16 \\ 2 & e_{1} \oplus e_{2} \oplus e_{4} & - & e_{2} \oplus e_{3} & - & |b|=1 / 2 \\ 3 & - & e_{1} \oplus e_{3} \oplus e_{4} & - & e_{4} \oplus e_{5} \oplus e_{7} & |b| \leq 1 / 128 \\ 4 & e_{1} \oplus e_{3} \oplus e_{4} & - & e_{4} \oplus e_{5} \oplus e_{7} & - & |b|=1 / 2\end{array}$

Nevertheless, there are complex interactions between the individual steps of $\mathrm{RC} 2$ and these often provide unintuitive results. In particular we have discovered cases where adding a non-trivial approximation to an existing approximation actually boosts the absolute value of the bias. (Such an example can be found in step 3 above when the subkeys in all rounds are set to zero.) Under such circumstances the true effectiveness of linear cryptanalysis in attacking RC2 has to remain an open problem.

\section{Conclusions}

In this paper we have described the block cipher RC2. While the cipher is perhaps slower than other alternatives available today, it does appear to offer effective resistance to differential cryptanalysis. Our attempts to apply linear cryptanalysis to RC2 have provided some intriguing insights, but are as yet insufficient to 
determine the actual resistance of $\mathrm{RC} 2$ to linear cryptanalysis; this remains an open problem. It is important that $\mathrm{RC} 2$ continues to come under close scrutiny from the cryptanalytic community. 


\section{References}

1. E. Biham and A. Shamir. Differential Cryptanalysis of the Data Encryption Standard. Springer-Verlag, New York, 1993.

2. J. Borst, L.R. Knudsen, and V. Rijmen. Two attacks on reduced IDEA. In W. Fumy, editor, Advances in Cryptology - Eurocrypt '97, volume 1233 of Lecture Notes in Computer Science, pages 1-13, 1997. Springer Verlag.

3. A. Biryukov and E. Kushilevitz. Improved cryptanalysis of RC5. Preprint.

4. M. Blaze and B. Schneier. The MacGuffin block cipher algorithm. In B. Preneel, editor, Fast Software Encryption, volume 1008 of Lecture Notes in Computer Science, pages 97-110, 1995. Springer Verlag.

5. S. Dusse, P. Hoffman, B. Ramsdell, L. Lundblade, and L. Repka. S/MIME Message Specification. September 23, 1997. Available from http://www.imc.org/draftdusse-smime-msg.

6. B.S. Kaliski and Y.L. Yin. On differential and linear cryptanalysis of the RC5 encryption algorithm. In D. Coppersmith, editor, Advances in Cryptology Crypto '95, volume 963 of Lecture Notes in Computer Science, pages 171-184, 1995. Springer Verlag.

7. L.R. Knudsen and W. Meier. Improved differential attacks on RC5. In N. Koblitz, editor, Advances in Cryptology - Crypto '96, volume 1109 of Lecture Notes in Computer Science, pages 216-228, 1996. Springer Verlag.

8. M. Matsui. Linear cryptanalysis method for DES cipher. In T. Helleseth, editor, Advances in Cryptology - Eurocrypt '93, volume 765 of Lecture Notes in Computer Science, pages 386-397, 1994. Springer-Verlag.

9. M. Matsui. The first experimental cryptanalysis of the Data Encryption Standard. In Y. Desmedt, editor, Advances in Cryptology - Crypto '94, volume 839 of Lecture Notes in Computer Science, pages 1-11, 1994. Springer-Verlag.

10. X. Lai, J. Massey and S. Murphy. Markov ciphers and differential cryptanalysis. In D. Davies, editor, Advances in Cryptology - Eurocrypt '91, volume 547 of Lecture Notes in Computer Science, pages 17-38, 1991. Springer Verlag.

11. National Institute of Standards and Technology (NIST). FIPS Publication 46-2: Data Encryption Standard. December 30, 1993.

12. R.L. Rivest. A Description of the $\mathrm{RC} 2^{\mathrm{TM}}$ Encryption Algorithm. File draft-rivest-rc2desc-00.txt available from ftp://ftp.ietf.org/internet-drafts/.

13. R.L. Rivest. The MD4 message digest algorithm. In A.J. Menezes and S.A. Vanstone, editors, Advances in Cryptology - Crypto '90, volume 537 of Lecture Notes in Computer Science, pages 303-311, 1991. Springer-Verlag.

14. R.L. Rivest. The RC5 encryption algorithm. In B. Preneel, editor, Fast Software Encryption, volume 1008 of Lecture Notes in Computer Science, pages 86-96, 1995. Springer Verlag. 


\section{Appendix}

The substitution table PITABLE specified in hexadecimal notation for input byte ab.

\begin{tabular}{|c|c|}
\hline a* & $* \mathrm{~b}$ \\
\hline & $\begin{array}{llllllllllllllll}0 & 1 & 2 & 3 & 4 & 5 & 6 & 7 & 8 & 9 & \mathrm{a} & \mathrm{b} & \mathrm{c} & \mathrm{d} & \mathrm{e} & \mathrm{f}\end{array}$ \\
\hline 00: & d9 78 f9 c4 19 dd b5 ed 28 e 9 fd $794 a$ a0 d8 $9 d$ \\
\hline 10: & c6 7 e 37832 2b 76538 e 62 4c $6488448 \mathrm{~b} \mathrm{fb} \mathrm{a2}$ \\
\hline 20: & 17 9a 59 f 587 b3 4 f 136145 dd $8 d 09817 d 32$ \\
\hline 30: & bd $8 f 40$ eb 86 b7 7b 0b f0 952122 5c $6 \mathrm{~b} 4$ e 82 \\
\hline 40: & 54 d 66593 ce 60 b2 1c 7356 c0 14 a 78 c f 1 dc \\
\hline 50: & 1275 ca 1 f $3 \mathrm{~b}$ be e 4 d 142 3d d 430 a 3 3c b 626 \\
\hline 60: & $6 \mathrm{f}$ bf $0 \mathrm{e}$ da 4669075727 f2 $1 \mathrm{~d} 9 \mathrm{~b}$ bc 944303 \\
\hline 70: & f8 11 c7 f 690 ef 3 e e 706 c 3 d5 2 f c8 66 e d \\
\hline 80: & 08 e 8 ea de 8052 ee $f 784$ aa 72 ac 35 4d $6 a$ \\
\hline 90: & 96 1a d2 71 5a 154974 4b 9f d0 5e 0418 a 4 ec \\
\hline $\mathrm{a} 0$ : & c2 e0 41 ee of $51 \mathrm{cb}$ cc 2491 af 50 a1 f 47039 \\
\hline b0: & 99 7c 3a 8523 b8 b4 7a fc 0236 5b 25559731 \\
\hline c0: & 2d 5 d fa 98 e3 8 a 92 ae 05 df $2910676 \mathrm{c}$ ba c9 \\
\hline do: & d3 00 e 6 cf e1 9e a8 2c 631601 3f 58 e2 89 as \\
\hline $\mathrm{e} 0$ : & Od 3834 1b ab 33 ff b0 bb 48 0c $5 f$ b9 b1 cd $2 e$ \\
\hline 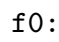 & c5 f3 db 47 e5 a5 9c 77 0a a6 2068 fe 7 f c1 ad \\
\hline
\end{tabular}

Test vectors for the RC2 encryption algorithm.

\begin{tabular}{|c|c|c|c|c|}
\hline $\begin{array}{c}\text { key } \\
\text { length } \\
\text { (bytes) }\end{array}$ & $\begin{array}{c}\text { effective } \\
\text { key } \\
\text { length } \\
\text { (bits) }\end{array}$ & key & plaintext & ciphertext \\
\hline 8 & 63 & 0000000000000000 & 0000000000000000 & ebb773f9 93278eff \\
\hline 8 & 64 & ffffffff ffffffff & ffffffff ffffffff & $278 \mathrm{~b} 27 \mathrm{e} 4 \quad 2 \mathrm{e} 2 \mathrm{f} 0 \mathrm{~d} 49$ \\
\hline 8 & 64 & 3000000000000000 & 1000000000000001 & $30649 \mathrm{edf} 9 \mathrm{be} 7 \mathrm{~d} 2 \mathrm{c} 2$ \\
\hline 1 & 64 & 88 & 0000000000000000 & 61a8a244 adacccf0 \\
\hline 7 & 64 & $88 \mathrm{bca} 90 \mathrm{e} 90875 \mathrm{a}$ & 0000000000000000 & $6 \mathrm{ccf} 4308$ 974c267f \\
\hline 16 & 64 & $\begin{array}{ll}88 b c a 90 e & 90875 a 7 f \\
0 f 79 c 384 & 627 b a f b 2\end{array}$ & 0000000000000000 & $1 \mathrm{a} 807 \mathrm{~d} 27 \quad 2 \mathrm{bbe} 5 \mathrm{db} 1$ \\
\hline 16 & 128 & $\begin{array}{l}\text { 88bca90e } 90875 a 7 f \\
\text { of79c384 }\end{array}$ & 0000000000000000 & $2269552 a$ b0f85ca6 \\
\hline 33 & 129 & 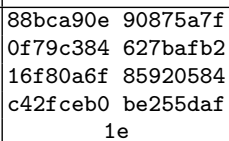 & 0000000000000000 & $5 \mathrm{~b} 78 \mathrm{~d} 3 \mathrm{a} 43 \mathrm{dfff} 1 \mathrm{f} 1$ \\
\hline
\end{tabular}

